\title{
The effect of inclination of channel on separation of an incompressible thermally and electrically conducting viscous binary fluid mixture in presence of strong magnetic field
}

\author{
B. R. Sharma ${ }^{1}$, R. N. Singh ${ }^{2}$, Rupam Kr.Gogoi ${ }^{3}$ \\ ${ }^{1}$ Dept.of Mathematics, Dibrugarh University, Dibrugarh-786004, India \\ ${ }^{2}$ Marwari Hindi High School, Jalukpara, Dibrugarh-786001, India \\ ${ }^{3}$ Dept. of Mathematics, Sibsagar College, Joysagar, Sivasagar-785665, India
}

\section{Email address:}

bishwaramsharma@yahoo.com (B.R. Sharma), nirojsingh@yahoo.co.in (R.N. Singh), gogoirupam@yahoo.com (R. K. Gogoi)

\section{To cite this article:}

B. R. Sharma, R. N. Singh, Rupam Kr.Gogoi. The Effect of Inclination of Channel on Separation of an Incompressible Thermally and Electrically Conducting Viscous Binary Fluid Mixture in Presence of Strong Magnetic Field. American Journal of Applied Mathematics. Vol. 2, No. 1, 2014, pp. 1-7. doi: 10.11648/j.ajam.20140201.11

\begin{abstract}
The effect of inclination of a channel on separation of a binary mixture of viscous incompressible thermally and electrically conducting Newtonian fluids in presence of a strong magnetic field perpendicular to the direction of flow is examined. The equations of motion, energy and concentration are solved analytically. It is found that the non-dimensional parameters viz, the baro-diffusion number, the Soret number, the product of Prandtl number and Eckert number, the Hartmann number, the electric parameter and the Magnetic Reynolds number affects the species separation significantly. The inclination of the channel has adverse effect on the rate of species separation while the intensity of the applied magnetic field enhanced the same.
\end{abstract}

Keywords: Binary Mixture, Baro-Diffusion, Magnetic Field

\section{Introduction}

Separation processes of components of a binary fluid mixture wherein one of the components is present in extremely small proportion are of much interest due to their application in science and technology. Besides environmental engineering applications, convection mass transfer alone contributes the backbone of many operations in chemical industry. Separation of isotopes from their naturally occurring mixture is one of such examples. It is well known that only one part of heavy water which is an isotope of water is found in 25,000 parts of water in normal occurrence (Arnikar[1], Rastogy et. al [2]) but is required for use as a (i) moderator in nuclear reactions for slowing down the neutrons, (ii) traces compound for studying the mechanism of many chemical reactions and (iii) heat transport medium i.e. a coolant in atomic power plant. Because of their small relative mass difference, isotopes of heavier molecules offer the greatest practical challenge in attempts to isolate the rarer component. Electromagnetic method of separation (Srivastava [3]) works only at relatively higher values of concentration.

Uranium is often grouped into a broader classification of contaminants particularly for drinking water, known as radionuclides. The most common radionuclides are found in drinking water that include uranium, radon and radium (Singh et. al [4] ). Drinking water containing radionuclides can cause adverse health effects. As a result of nonbiodegradable nature, the heavy metals including uranium accumulate in vital human organs and exert progressively growing toxic action [5]. Most notably, long term ingestion of uranium and some other heavy metals may increase the risk of kidney damage, cancer and cardiovascular disease (see Ref. [6,7]) whereas the environmental evidence suggests that the respiratory and reproductive system are also affected by uranium exposure as in Ref. [8]. Hence the public community water supplier must comply with the maximum contaminated limit (MCL) recommended by various National and International agencies like $15 \mathrm{ppb}$ in Hoo et. al. [9], $30 \mathrm{ppb}$ in [10], 9 ppb in [8] etc. The high concentration of radionuclides can be reduced to MCL by separating them from the water. The problem discussed 
here derives its application also in the basic fluid dynamics separation process to separate the rarer component of the different isotopes of heavier molecules where electromagnetic method of separation does not work.

In a binary fluid mixture the diffusion of individual species takes place by three mechanisms namely ordinary diffusion, pressure diffusion (baro-diffusion) and thermal diffusion.The diffusion flux $\mathbf{i}$ of lighter and rarer component is given by Landau and Lifshitz [11] as

$$
\mathbf{i}=-\rho \mathcal{D}\left[\nabla \mathrm{c}_{1}+\mathrm{k}_{\mathrm{p}} \nabla \mathrm{p}+\mathrm{k}_{\mathrm{T}} \nabla \mathrm{T}\right]
$$

The ordinary diffusion contribution to the mass flux is seen to depend in a complicated way on the concentration gradients of the component present in the mixture. The baro-diffusion indicates that there may be a net movement of the components in a mixture if there is a pressure gradient imposed on the system. An example of barodiffusion is the process of diffusion in the binary mixture of different kinds of gases present in the atmosphere. By reasons of variation of forces of gravity with height thereby causing a density gradient, different constituents of the atmosphere tend to separate out. The pressure gradient created by the gravity as well as the rotation of the earth separates various components of air. The tendency for a mixture to separate under a pressure gradient is very small but use is made of this effect in centrifuge separation in which tremendous pressure gradient is established. Thermal diffusion describes the tendency for species to diffuse under the influence of a temperature gradient. In many practical problems dealing with flows in porous media one encounters with a multiple component electrically conducting fluids e.g. molten fluids in the earth's crust, crude oil in the petroleum. It is customary to consider one of the components as solvent and the other components as solute. It is shown in Ref. Groot and Mazur [12] that if separation due to thermal diffusion occurs then it may even render an unstable system to stable one. This effect is also quite small, but devices can be arranged to produce very steep temperature gradients so that separations of mixtures are effected.

The problem of the flow of the fluid between two infinite parallel plates, one of which is stationary and the other is moving with a constant velocity, under the influence of a pressure gradient, has been worked out in Schlichting [13] and the corresponding problem of the heat transfer has been worked out in Pai [14]. Many authors have extended these problems in many different ways. De Groff [15] generalized Couette motion to include the case when the viscosity of the fluid depends on temperature. Kapur and Sukla [16] considered the flow of layers of different heights of an incompressible immiscible fluid between two parallel plates and found that whatever be the number of fluids and whatever be their heights, a unique maximum velocity exists. Shah [17] has discussed the effect of pressure gradient and temperature gradient on separation of a binary mixture of incompressible viscous fluids confined between two parallel plates and found that the effect of temperature gradient and pressure gradient is to gather the lighter component of the fluid mixture near the stationary wall and throw away the heavier component to the moving wall.

To exhibit the effect of the inclination of a channel on separation of a binary fluid mixture, we discuss in this paper the mass diffusion of a binary mixture of incompressible, viscous thermally and electrically conducting fluids of unequal molecular weights in an inclined channel in presence of strong magnetic field.

\section{Governing Equations and Boundary Conditions}

We consider here the case when one of the components of the binary mixture of incompressible thermally and electrically conducting viscous fluids is present in small quantity, hence the density and viscosity of the mixture is independent of the distribution of the components. The concentration $c_{2}$ of heavierand more abundant component is given by $c_{2}=1-c_{1}$. The flow problem of the binary mixture is identical to that of a single fluid, but the velocity is to be understood as the mass average velocity $\mathrm{V}=$ $\left(\rho_{1} V_{1}+\rho_{2} V_{2}\right) / \rho$ and the density $\rho=\rho_{1}+\rho_{2}$, where the subscripts 1 and 2 denote the rarer and the more abundant components respectively. The equation of continuity and the equation of motion of an incompressible fluid in steady case are respectively,

$$
\nabla \cdot \mathrm{V}=0
$$

and

$$
\rho(\mathrm{V} . \nabla) \mathrm{V}=-\nabla \mathrm{p}+\rho \mathrm{F}+\mu \nabla^{2} \mathrm{~V}+\mathrm{J} \times \mathrm{B}
$$

In steady motion the Maxwell equations are given by

$$
\begin{aligned}
& \operatorname{curlH}=4 \pi \\
& \operatorname{curlE}=0
\end{aligned}
$$

and

$$
\operatorname{divH}=0
$$

It is well known that for most of the fluids used in engineering applications collision frequency exceeds the cyclotron frequency for electrons. As the Hall current factor is ratio of the cyclotron frequency to the collision frequency, the Hall current is very small and so we have neglected it in our discussion. Consequently Ohm's law is given by

$$
\mathrm{J}=\sigma[\mathrm{E}+\mathrm{V} \times \mathrm{B}]
$$

Where

$$
\mathrm{B}=\mu_{\mathrm{e}} \mathrm{H}
$$

The magnetic induction equation is given by

$$
\frac{\partial \mathrm{B}}{\partial \mathrm{t}}=\nabla \times(\mathrm{V} \times \mathrm{B})+\eta_{\mathrm{m}} \nabla^{2} \mathrm{~B}
$$

The energy equation in steady case is given by 


$$
\mu \mathrm{c}_{\mathrm{p}} \mathrm{V} . \nabla \mathrm{T}=\kappa \nabla^{2} \mathrm{~T}+\mu \varphi+\frac{\mathrm{J}^{2}}{\sigma}
$$

where the last term $\frac{\mathrm{J}^{2}}{\sigma}$ represents heat due to electrical resistive dissipation.

The equation of species conservation of the first component is given by (see Landau and Lifshitz [11])

$$
\rho(\mathrm{V} . \nabla) \mathrm{c}_{1}=-\nabla . \mathrm{i}
$$

wherei is given by (1) . The co-efficients $\mathrm{k}_{\mathrm{p}}$ and $\mathrm{k}_{\mathrm{T}}$ may be determined from the thermodynamic properties alone. Landau and Lifshitz [11] have given the explicit expression for the baro diffusion ratio $\mathrm{k}_{\mathrm{p}}$ as

$$
\mathrm{k}_{\mathrm{p}}=\left(\mathrm{m}_{2}-\mathrm{m}_{1}\right)\left[\frac{\mathrm{c}_{1}}{\mathrm{~m}_{1}}+\frac{\mathrm{c}_{2}}{\mathrm{~m}_{2}}\right] \frac{\mathrm{c}_{1} \mathrm{c}_{2}}{\mathrm{p}_{\infty}}
$$

Since $c_{2}=1-c_{1}$ and we have assumed $c_{1}$ to be very small so $c_{1}^{2}$ may be neglected and hence (12) becomes

$$
\mathrm{k}_{\mathrm{p}}=\frac{\left(\mathrm{m}_{2}-\mathrm{m}_{1}\right) \mathrm{c}_{1}}{\mathrm{~m}_{2} \mathrm{p}_{\infty}}=\mathrm{Ac}_{1}
$$

where

$$
\mathrm{A}=\frac{\mathrm{m}_{2}-\mathrm{m}_{1}}{\mathrm{~m}_{2} \mathrm{p}_{\infty}}
$$

The expression $\mathrm{k}_{\mathrm{T}}$ has been suggested Hurl and Jakeman[18] as

$$
\mathrm{k}_{\mathrm{T}}=\mathrm{s}_{\mathrm{T}} \mathrm{c}_{1} \mathrm{c}_{2}
$$

For small value of $c_{1}$ (since $c_{2}=1-c_{1}$, and we have assumed $c_{1}$ to be very small, so $c_{1}^{2}$ may be neglected) (15) becomes

$$
\mathrm{k}_{\mathrm{T}}=\mathrm{s}_{\mathrm{T}} \mathrm{c}_{1}
$$

Substituting the expression for $\mathbf{i}$ from (1), $\mathrm{k}_{\mathrm{p}}$ from (13) and $\mathrm{k}_{\mathrm{T}}$ from (16) in (11) we get the equation for $\mathrm{c}_{1}$ as

$$
(\mathrm{V} . \nabla) \mathrm{c}_{1}=\mathcal{D}\left[\nabla^{2} \mathrm{c}_{1}+\mathrm{A} \nabla \cdot\left(\mathrm{c}_{1} \nabla \mathrm{p}\right)+\mathrm{s}_{\mathrm{T}} \nabla \cdot\left(\mathrm{c}_{1} \nabla \mathrm{T}\right)\right]
$$

The boundary conditions for velocity are $\mathrm{V}=0$ at solid surfaces since the surfaces are stationary. The boundary conditions for temperature, $\mathrm{T}=\mathrm{T}_{0}$ at both plates. The boundary conditions for magnetic field are $b=0$ at both the plates. The boundary conditions for the concentration $c_{1}$ are different in different cases. At the surface of a body insoluble in the fluid mixture the total mass flux as well as the individual species flux normal to the surface should vanish (Srivastav [20]) i.e.

$$
\rho c_{1} V \cdot n+i . n=0
$$

where $\mathrm{n}$ is the unit normal drawn at the solid surface directed outwards.

Substituting the expression for i from (1) into in (18), we get

$$
\rho c_{1} V \cdot n-\rho \mathcal{D}\left[\nabla c_{1} \cdot n+k_{p} \nabla p \cdot n+k_{T} \nabla T \cdot n\right]=0
$$

If however, there is diffusion from a body that dissolves in the fluid, equilibrium is rapidly established near its surface and the concentration in the fluid adjoining the plates in this case is the saturation concentration $\mathrm{c}_{0}$ (say); the diffusion out of this layer takes more slowly than the process of solution. The boundary condition at such surface is, therefore

$$
\mathrm{c}=\mathrm{c}_{0}
$$

\section{Formulation of the Problem}

In this problem we consider that a steady two dimensional motion of an incompressible thermally and electrically conducting viscous binary fluid mixture flowing through an inclined channel between two parallel flat plates which are at distance ' $2 \mathrm{~h}$ ' apart under the influence of a strong uniform transverse magnetic field. It has been assumed that the $\mathrm{x}$-axis is parallel to mid-way between the two plates, the y-axis perpendicular to it. Both the plates are maintained at uniform constant temperatures $T_{0}$. A strong uniform magnetic field of strength $B_{0}$ is applied in transverse direction, and therefore the induced magnetic field $b_{x}$ is developed in $x$-direction. As we consider here that the flow is along $\mathrm{x}$-axis, so the flow depends only on $y$ and the velocity vector is of the form $(u(y), 0,0)$. The above geometry suggests that the magnetic field is of the form $\left(b_{x}, B_{0}, 0\right)$ and electric field is of the form $\left(0,0, E_{z}\right)$. Here it is assumed that both the plates are at rest. The upper plate is considered to be impervious and the lower one at a constant concentration.

Under these assumption, the governing equations (3), (9), (10), and (17) for the steady flow of a binary mixture of incompressible thermally and electrically conducting viscous fluids between two non-conducting parallel plates in presence of magnetic field become respectively as

$$
\begin{gathered}
\mu \frac{d^{2} u}{d y^{2}}=-\rho g \sin \theta+\sigma\left(E_{z}+U B_{0}\right) B_{0} \\
\frac{d p}{d y}=-\rho g \cos \theta+\sigma\left(E_{z}+u B_{0}\right) b_{x} \\
\kappa \frac{d^{2} T}{d y^{2}}+\mu\left(\frac{d u}{d y}\right)^{2}+\sigma\left(E_{z}+u B_{0}\right)^{2}
\end{gathered}
$$

and

$$
\frac{d^{2} c_{1}}{d y^{2}}+A \frac{d}{d y}\left(c_{1} \frac{d p}{d y}\right)+S_{T} \frac{d}{d y}\left(c_{1} \frac{d T}{d y}\right)=0
$$

with the boundary conditions: 


$$
\begin{aligned}
& u=0, b_{x}=0, T=T_{0}, \frac{d c_{1}}{d y}+A c_{1} \frac{d p}{d y}+S_{T} c_{1} \frac{d T}{d y}=0 \\
& \text { at } y=h \\
& u=0, b_{x}=0, T=T_{0}, c_{1}=c_{0} \\
& \text { at } y=-h
\end{aligned}
$$

To write the system of equation in a dimensionless form we use the following variables transformations:

$$
f(\eta)=\frac{u}{\rho g h^{2} / \mu}, \eta=\frac{y}{h}, p=\rho g h p^{*}, T^{*}=\frac{T-T_{0}}{T_{0}}, b=\frac{b_{x}}{B_{0}}, c=\frac{c_{1}}{c_{0}} .
$$

Using these transformations, equations (21)-(24) take the form as:

$$
\begin{gathered}
\frac{d^{2} f}{d \eta^{2}}-M^{2} f=M^{2} R_{E}-\sin \psi \\
\frac{d p^{*}}{d \eta}=-\cos \psi+\left(f+R_{E}\right) M^{2} b \\
\frac{d^{2} b}{d \eta^{2}}+R_{m} \frac{d f}{d \eta}=0 \\
\frac{d^{2} T^{*}}{d \eta^{2}}+P_{r} E_{c}\left(\frac{d f}{d \eta}\right)^{2}+P_{r} E_{c} M^{2}\left(R_{E}+f\right)^{2}=0
\end{gathered}
$$

and

$$
\frac{d}{d \eta}\left\{\frac{d c}{d \eta}+B_{d} c \frac{d p^{*}}{d \eta}+t_{d} c \frac{d T^{*}}{d \eta}\right\}=0
$$

where $M=h B_{0} \sqrt{\sigma / \mu}, R_{E}=\frac{E_{z} \mu}{\rho g h^{2} B_{0}}, R_{m}=\frac{\rho g h^{3}}{\mu \eta_{m}}, P_{r} E_{c}=\frac{\rho^{2} g^{2} h^{4}}{\mu \kappa T_{0}}$, $B_{d}=A \rho g h$ and $t_{d}=T_{0} S_{T}$. The boundary conditions (25) on velocity, magnetic field, temperature and concentration in terms of dimensionless quantities are

$$
\begin{aligned}
& f=0, b=0, \quad T^{*}=0, \frac{d c}{d \eta}+B_{d} c \frac{d p^{*}}{d \eta}+t_{d} c \frac{d T^{*}}{d \eta}=0 \quad \text { at } \quad \eta=1 \\
& f=0, b=0, T^{*}=0, c=1 \quad \text { at } \eta=-1
\end{aligned}
$$

\section{Solution of the Problem}

The exact solutions of the equations (27)-(31) subject to boundary conditions (32) are obtained and are given by

$$
\begin{aligned}
f= & A_{0}\left(1-\frac{\cosh M \eta}{\cosh M}\right) \\
\mathrm{T}^{*}= & \frac{\mathrm{P}_{\mathrm{r}} \mathrm{E}_{\mathrm{c}} \mathrm{A}_{0}^{2}}{4 \cosh ^{2} \mathrm{M}}(\cosh 2 \mathrm{M}-\cosh 2 \mathrm{M} \eta) \\
& +\frac{1}{2} \mathrm{P}_{\mathrm{r}} \mathrm{E}_{\mathrm{c}} \mathrm{M}^{2}\left(\mathrm{R}_{\mathrm{E}}+\mathrm{A}_{0}\right)^{2}\left(1-\eta^{2}\right) \\
& -\frac{2 \mathrm{P}_{\mathrm{r}} \mathrm{E}_{\mathrm{c}} \mathrm{A}_{0}}{\cosh \mathrm{M}}\left(\mathrm{R}_{\mathrm{E}}+\mathrm{A}_{0}\right)(\cosh \mathrm{M}-\cosh \mathrm{M \eta})
\end{aligned}
$$

$$
b=\frac{A_{0} R_{m}}{M \cosh M}(\sinh M \eta-\eta \sinh M)
$$

$$
\begin{aligned}
p^{*}(\eta)-p^{*}(-1)=-(\eta+1) \cos \psi+\frac{A_{0} R_{m}}{\cosh M} & \\
& {\left[\left(A_{0}+R_{E}\right)(\cosh M \eta-\cosh M)-\frac{1}{2} M\left(A_{0}+R_{E}\right)\left(\eta^{2}-1\right) \sinh M\right.} \\
- & \frac{A_{0}}{4 \cosh M}(\cosh 2 M \eta-\cosh 2 M)+ \\
& \left.\frac{A_{0} \sinh M}{\cosh M}\left\{(\eta \sinh M \eta-\sinh M)-\frac{1}{M}(\cosh M \eta-\cosh M)\right\}\right]
\end{aligned}
$$

and

$$
c=\exp \left[-B_{d}\left\{p^{*}(\eta)-p^{*}(-1)\right\}-t_{d} T^{*}(\eta)\right\rfloor
$$

where $A_{0}=\frac{1}{M^{2}} \sin \psi-R_{E}$

\section{Discussion and Conclusion}

In absence of magnetic field the Eq. (37) produces a singular solution. So, putting $\mathrm{M}=0$ in equations (27)-(31) and solving under the boundary conditions (32), we get

$$
\left.c(\eta)\right|_{M=0}=\exp \left[-\frac{1}{12} P_{r} E_{c} t_{d}\left(1-\eta^{4}\right) \sin ^{2} \psi+B_{d}(1+\eta) \cos \psi\right]
$$

If we put $t_{d}=0$ and $B_{d}=0$ in equations (37) and (38), we get $c(\eta)=1$ for all values of $\eta$. From this it can be concluded that the separation of species ceases to take place if we neglect the effects of temperature gradient and pressure gradient. The magnetic field present in the medium has no direct effect on the process of separation. It influences the temperature gradient which consequently affects the process of separation of the constituents of the mixture.

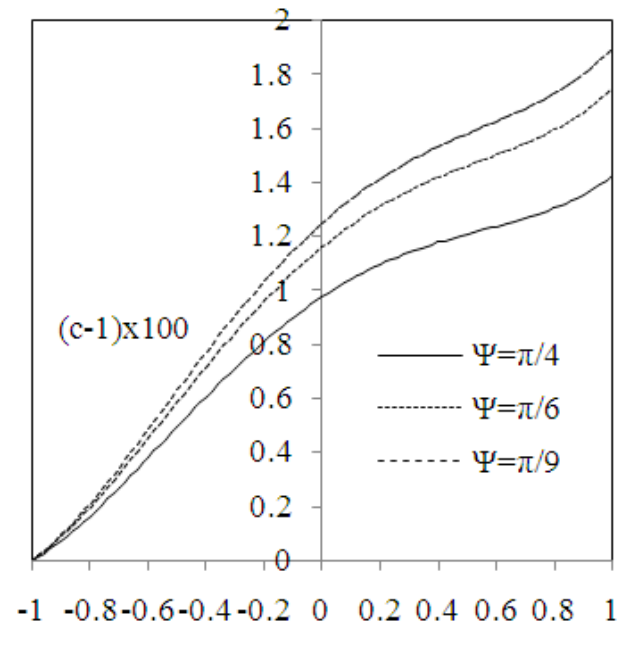

$\eta$

Fig 1.The graphs of the concentration profile $c(\eta)$ against the width of the channel taking $M=0.5, P_{r} E_{c}=0.01, B_{d}=0.01, t_{d}=0.01, R_{E}=10, R_{m}=2$ for the various values $\psi$.

The Fig. 1 shows the plot of concentration of the lighter and rarer component of the binary fluid mixture for the 
various values of $\psi=\pi / 9, \pi / 6, \pi / 4$ by taking $\mathrm{t}_{\mathrm{d}}=0.01, \mathrm{~B}_{\mathrm{d}}=0.01$, $R_{E}=10, M=0.5, R_{m}=2, P_{r} E_{c}=0.01$ and it is found that the inclination of the channel has adverse effect on the separation of the species of the lighter component of the binary fluid mixture i.e. decease in the value of $\psi$ increases the species separation.

The Fig. 2 shows the plot of concentration of the lighter and rarer component of the binary fluid mixture for various values of the parameters $t_{d}=0.001,0.005,0.01$ by taking $\psi=$ $\pi / 9, \mathrm{~B}_{\mathrm{d}}=0.01, \mathrm{R}_{\mathrm{E}}=10, \mathrm{M}=0.5, \mathrm{R}_{\mathrm{m}}=2, \mathrm{P}_{\mathrm{r}} \mathrm{E}_{\mathrm{c}}=0.01$ and found that the species separation increases with the decrease in the value of $t_{d}$. The graph reveals that the separation is more in between the plates.

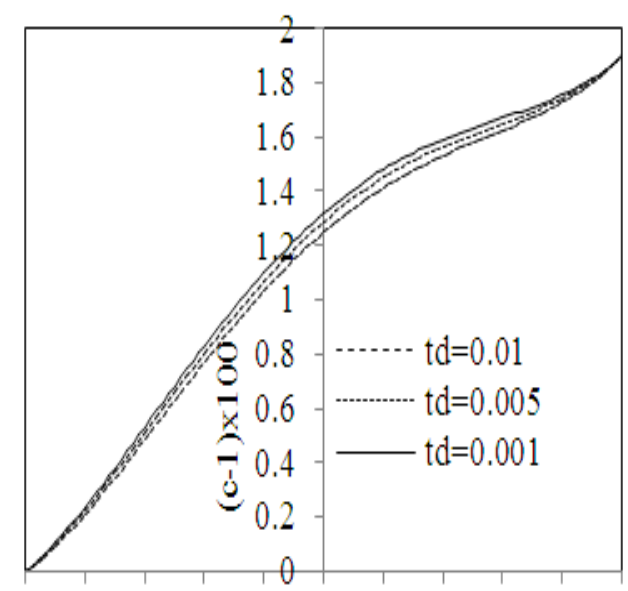

$\cdot 1 \quad-0.8-0.6-0.4-0.2 \quad 0 \quad 0.2 \quad 0.4 \quad 0.6 \quad 0.8 \quad 1$

\section{$\eta$}

Fig 2. The graphs of the concentration profile $c(\eta)$ against the width of the channel taking $\psi=\pi / 9, M=0.5, P_{r} E_{c}=0.01, B_{d}=0.01, R_{E}=10, R_{m}=2$ for the various values of Soret number.

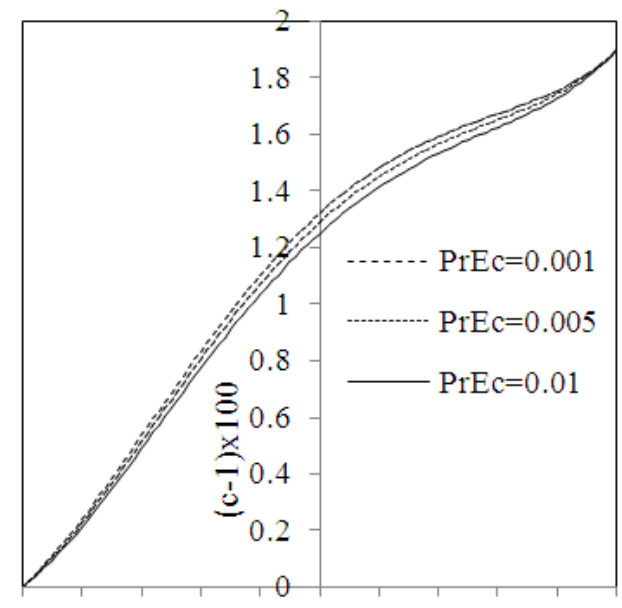

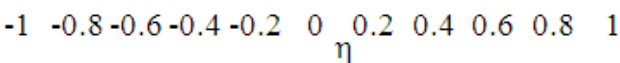

Fig 3. The graphs of the concentration profile $c(\eta)$ against the width of the channel taking $\psi=\pi / 9, M=0.5, R_{E}=10, R_{m}=2, B_{d}=0.01, t_{d}=0.01$ for the various values of the product of Prandtl's number and Eckert's number.

The Fig. 3 shows the plot of concentration of the lighter and rarer component of the binary fluid mixture for various values of $\mathrm{P}_{\mathrm{r}} \mathrm{E}_{\mathrm{c}}=0.001,0.005,0.01$ by taking $\mathrm{t}_{\mathrm{d}}=0.01$, $B_{d}=0.01, R_{E}=10, M=0.5, R_{m}=2, \psi=\pi / 9$. It is found that the effect of the product of the Prandtl number and the Eckert number is similar to that of the thermal diffusion number.

The Fig. 4 shows the plot of concentration of the lighter and rarer component of the binary fluid mixture for the various values of electric parameter $R_{E}=8,10,12$ by taking $\mathrm{t}_{\mathrm{d}}=0.01, \mathrm{~B}_{\mathrm{d}}=0.01, \psi=\pi / 9, \mathrm{M}=0.5, \mathrm{R}_{\mathrm{m}}=2, \mathrm{P}_{\mathrm{r}} \mathrm{E}_{\mathrm{c}}=0.01$ and found that the effect of electric parameter is to through the heavier component away from the lower plate at $\eta=-1$ i.e. an increase in the value of the electric parameter increases of the concentration of the lighter component of the binary fluid mixture.

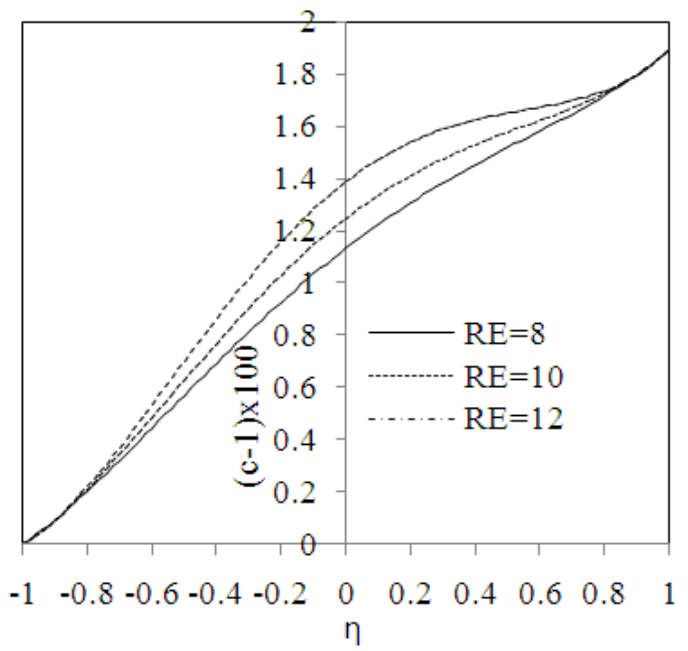

Fig 4. The graphs of the concentration profile $c(\eta)$ against the width of the channel taking $\psi=\pi / 9, M=0.5, R_{m}=2, \operatorname{Pr} E_{c}=0.01, B_{d}=0.01, t_{d}=0.01$ for various values of the electric field parameter.

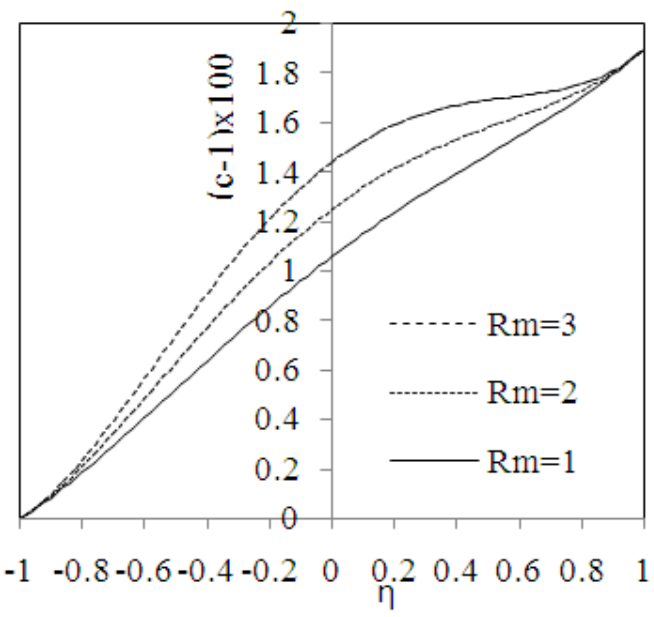

Fig 5.The graphs of the concentration profile $c(\eta)$ against the width of the channel taking $\psi=\pi / 9, M=0.5, R_{E}=10, P_{r} E_{c}=0.01, t_{d}=0.01, B_{d}=0.01$ for the various values of the magnetic Reynolds number.

The Fig. 5 shows the plot of concentration of the lighter and rarer component of the binary fluid mixture for various values of the magnetic Reynolds number $R_{m}=1,2,3$ by taking $\mathrm{t}_{\mathrm{d}}=0.01, \mathrm{~B}_{\mathrm{d}}=0.01, \mathrm{R}_{\mathrm{E}}=10, \mathrm{M}=0.5, \psi=\pi / 9, \mathrm{P}_{\mathrm{r}} \mathrm{E}_{\mathrm{c}}=0.01$ and found that the magnetic Reynolds number favour 
thespecies separationof the lighter component of the binary fluid mixture i.e. the increase of the value of $R_{m}$ increases the effect of species separation of the lighter component.

The Fig. 6 shows the plot of concentration of the lighter and rarer component of the binary fluid mixture for various values of $\mathrm{M}=0,0.5,1,1.5$, 2by taking $\mathrm{t}_{\mathrm{d}}=0.01, \mathrm{~B}_{\mathrm{d}}=0.01$, $\mathrm{R}_{\mathrm{E}}=10, \psi=\pi / 9, \mathrm{R}_{\mathrm{m}}=2, \mathrm{P}_{\mathrm{r}} \mathrm{E}_{\mathrm{c}}=0.01$. The graph reveals that the applied constant magnetic field normal to the plates favours the species separation of the lighter component of the binary fluid mixture i.e. an increase in the values $M$ increases the effect of species separation of the lighter component. This effect is found only in between the plates and is maximum in the central part of the channel.

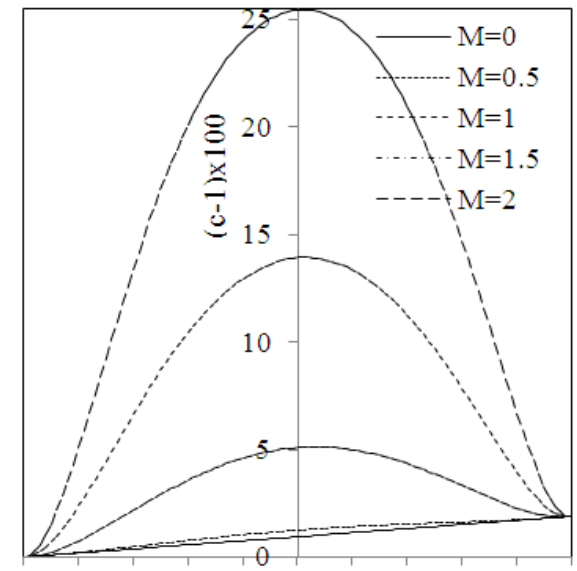

$\begin{array}{lllllllllll}-1 & -0.8 & -0.6 & -0.4 & -0.2 & 0 & 0.2 & 0.4 & 0.6 & 0.8 & 1\end{array}$

Fig6. The graphs of the concentration profile $c(\eta)$ against the width of the channel taking $\psi=\pi / 9, R_{E}=10, R_{m}=2, \operatorname{Pr} E_{c}=0.01, B_{d}=0.01, t_{d}=0.01$ for the various values of the Hartmann number.

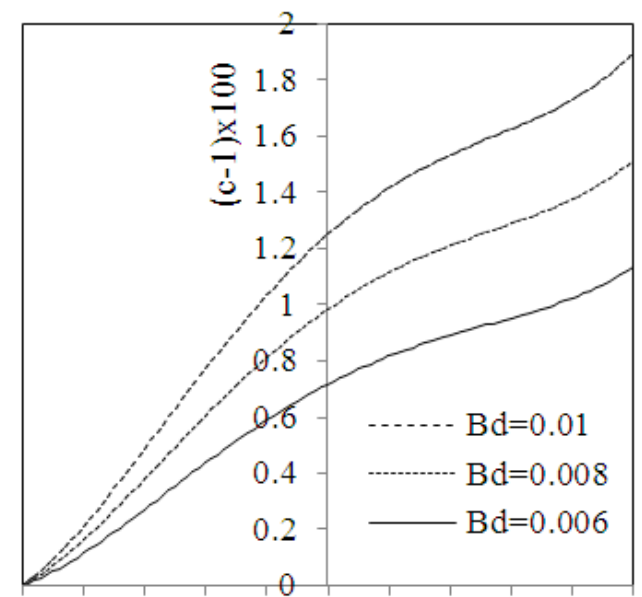

$\begin{array}{lllllllll}-1 & -0.8-0.6-0.4-0.2 & 0 & 0.2 & 0.4 & 0.6 & 0.8 & 1\end{array}$

Fig 7.The graphs of the concentration profile $c(\eta)$ against the width of the channel taking $\psi=\pi / 9, M=0.5, P_{r} E_{c}=0.01, t_{d}=0.01, R_{E}=10, R_{m}=2$ for the various values of baro-diffusion number.

The Fig. 7 shows the plot of concentration of the lighter and rarer component of the binary fluid mixture for various values of the baro-diffusion number $\mathrm{B}_{\mathrm{d}}=0.006,0.008,0.01$ by taking $t_{d}=0.01, \psi=\pi / 9, R_{E}=10, M=0.5, R_{m}=2, P_{r} E_{c}=0.01$ and found that the effect of baro-diffusion is to through the heavier component away from the lower plate at $\eta=-1$ i.e. an increase in the value of baro-diffusion coefficient increases the concentration of the lighter component of the binary fluid mixture. The concentration of the lighter and rarer component is found to be maximum at the upper plate.

From the above discussion it can be concluded that the process of separation of the species of the binary fluid mixture can be enhanced, by decreasing the inclination of the channel, the thermal diffusion number, Prandtl number, the Eckert number and by increasing the intensity of the applied magnetic field, the baro-diffusion coefficient, magnetic Reynolds number, the electric parameter.

\section{List of Symbols}

B Magnetic inductance vector

$\mathrm{B}_{0} \quad$ Uniform applied magnetic field

$\mathrm{B}_{\mathrm{d}} \quad$ Barodiffusion number

$\mathrm{b}_{\mathrm{x}} \quad$ Induced magnetic field along the plate

c Concentration

Concentration of the first component of the binary mixture

$c_{2}$ Concentration of the second component of the binary mixture

$c_{p} \quad$ Specific heat at constant pressure

Concentration of lighter and rarer component of the

$\mathrm{c}_{0}$ binary fluid mixture at upper plate

$D \quad$ Diffusion coefficient

E Electric field vector

$\mathrm{E}_{\mathrm{c}} \quad$ Eckert number

$\mathrm{E}_{\mathrm{z}} \quad$ Component of electric field along z-direction

$\mathrm{F} \quad$ Body force per unit mass

$\mathrm{H}$ Magnetic field vector

$\mathrm{h}$ half width of the channel

i Diffusion flux density vector

J Current density vector

$\mathrm{k}_{\mathrm{p}} \quad$ Barodiffusion ratio

$\mathrm{k}_{\mathrm{T}} \quad$ Thermal diffusion ratio

M Hartmann number

$\mathrm{m}_{1} \quad$ Mass of first kind of the particle

$\mathrm{m}_{2} \quad$ Mass of second kind of the particle

$\mathrm{n} \quad$ Unit vector drawn perpendicular to the plates

$\mathrm{p}$ Pressure

$\mathrm{P}_{\mathrm{m}} \quad$ Magnetic Prandtl number

$\mathrm{p}_{\infty} \quad$ Working pressure of the medium

$\mathrm{P}_{\mathrm{r}} \quad$ Prandtl number 
R Reynolds number

$\mathrm{R}_{\mathrm{E}} \quad$ Electric field parameter

$\mathrm{R}_{\mathrm{m}} \quad$ Magnetic Reynolds number

$\mathrm{S}_{\mathrm{T}} \quad$ Soret coefficient

$\mathrm{T}$ Temperature, $\mathrm{T}_{0}$, temperature of the plates

$t_{d} \quad$ Soret number

$\mathrm{u} \quad$ Velocity along $\mathrm{x}$-direction

$\mathrm{V}$ Average velocity

$\mathrm{V}_{1} \quad$ Velocity of rarer and lighter component

$\mathrm{V}_{2} \quad$ Velocity of more abundant component

$\mathrm{x}$

Co-ordinate measuring the distance parallel to the plate

Co-ordinate measuring the distance perpendicular to the plate

Co-ordinate measuring the distance perpendicular to both $\mathrm{x}$-axis and $\mathrm{y}$-axis

\section{Greek Symbols}

$\phi \quad$ Heat due to viscous dissipation

$\eta \quad$ Non-dimensional variable measuring the distance

$\eta$ perpendicular to the plate

Coefficient of magnetic viscosity or magnetic

$\eta_{\mathrm{m}}$ diffusivity

$\kappa \quad$ Thermal conductivity

$\mu \quad$ Coefficient of viscosity

$\mu_{\mathrm{e}} \quad$ Magnetic permeability

$v \quad$ Coefficient of kinematic viscosity

$\rho \quad$ Density of binary fluid mixture

$\rho_{1} \quad$ Density of the first component of the binary mixture

Density of the second component of the binary

$\rho_{2}$ mixture

$\sigma \quad$ Electrical conductivity

$\psi \quad$ Inclination of the channel to the horizontal

\section{References}

[1] Arnikar HJ (1963) Essentials of Nuclear Chemistry. New age international ( P) limited, London

[2] Rastogy RP, Nath N, Singh NB (1992) Modern Inorganic Chemistry.united Book Depot,allahbad, India

[3] Srivastava AC (1992) Mass Diffusion in a Binary Mixture of Viscous Fluids. ProclNatlAcadSci69 (A2):103-117

[4] Singh H, Singh J, Bajwa BS (2009) Uranium Concentration in the Drinking water Sample Using the SSNTDs. Indian J Phys 83(7):1039 - 1044

[5] ASTDR Agency for Toxic Substances and DisesaseRegistry( 1999), Atalanta, GA

[6] Kumaresan M. Riyazuddin P (1999) Chemical Speciation of Trace Metals( Review). Res Chem Environ 3(4) : 59-79

[7] Charles M (2001)UNSCEAE Report 2000: Sourcesc and effects of Ionizing Radiation . J RadiolProt 21:83-85

[8] WHO Guidelines for Drinking WaterQuality (2004), Third Edition

[9] Hoo LS, Samatl A, Othman MR (2004) The Crucial Concept Posed y Aquatic Organism in Assessing the Lotic System Water Quality : A review. Res J Chem Environ 8 (2):24-30

[10] US EPA ( 2003) Current Drinking Water Standards ,pp 1-12

[11] Landau LD, Lifshitz EM (1960) Electrodynamics of Continuous Media. Pergamon Press, New York

[12] De Groot SR, Mazur P, Mazur S (1962) Non - Equilibrium Thermodynamics. North Holland Publishing Co, Amsterdam

[13] Schlichting, H. (1951), Boundary Layer Theory, 1st German edition, pp.70-71 \& 292.

[14] Pai, S. I. (1956) Viscous Flow theory, 1- Laminar flow, D. Van Nostrand Company, Inc., London

[15] De Groff, H. M. (1956), Journal of Applied Sciences, Vol. 23, pp. 395-396.

[16] Kapur, J. N. and Sukla, J. B. (1964), Applied Science Research, Section A, Vol. 8, pp. 55-60.

[17] Shah, N. A. (1996), Ph. D. Thesis, Submitted to the Dibrugarh University, Dibrugarh, Assam, India

[18] Jakeman, E.,Hurle, D. T. ,Soret-driven thermo-solutal convection, J. Fluid Mech., 47(4) (1971),667-687. 